\title{
Efeito da aplicação dos extratos vegetais na pré-colheita em uva cultivar Bordô
}

\section{Effect of the application of vegetable extracts in pre-harvest in the Bordô grape cultivar}

\author{
Thiécla Katiane Rosales Silva*, Cacilda Márcia Duarte Rios Faria, Aline José Maia \\ e Renato Vasconcelos Botelho
}

Universidade Estadual do Centro-oeste/ UNICENTRO, Departamento de Nutrição. Guarapuava-Paraná-Brasil

(*Email: thieclarosales@gmail.com)

http://dx.doi.org/10.19084/RCA16070

Recebido/received: 2016.06.07

Recebido em versão revista/received in revised form: 2016.10.31

Aceite/accepted: 2016.11 .08

\section{R E S U M O}

Objetivou-se avaliar a eficácia da aplicação de diferentes extratos vegetais na qualidade pós-colheita em uvas orgânicas de cultivar Bordô (Vitis labrusca). Para a preparação dos extratos foram utilizadas as folhas secas de gervão (Stachytarphetta cayenensis) e de capim-limão (Cymbopogon citratus); folhas frescas de pau d'alho (Gallesia integrifolia) e resíduo de vinificação (pó) proveniente de uva cultivar Isabel (Vitis labrusca). Cada extrato foi aplicado na concentração de $12 \%$ diretamente aos cachos 10 dias antes da colheita e uma parcela de cachos recebeu além da aplicação no campo uma nova aplicação na pós-colheita. O delineamento experimental foi blocos ao acaso, em esquema fatorial com $5 \times 2$ (cinco tratamentos e aplicação pré-colheita e pós-colheita) com quatro repetições. Foram avaliados a cor, pH, acidez titulável, índice de maturação, compostos fenólicos, antocianinas e análise sensorial. Verificou-se que os extratos foram eficazes para manter acidez titulável, sólidos solúveis, índice de maturação e a análise sensorial. Contudo, em bagas com aplicação do extrato de capim-limão aplicado em pré e pós-colheita observou-se valores adequados para as variáveis $\mathrm{pH}$, croma, antocianinas, apresentando uma coloração mais intensa. O extrato de gervão aplicado pré e pós-colheita manteve um conteúdo maior de fenólicos quando comparado à aplicação somente em pré-colheita. Indica-se a aplicação pré e pós-colheita dos extratos de capim-limão e gervão para a qualidade no armazenamento em uvas.

Palavras-Chave: armazenamento, tratamento alternativo, Vitis labrusca.

\begin{abstract}
A B S T R A C T
This study aimed to evaluate the effectiveness of the application of different plant extracts in the postharvest quality in organic Bordô grapes cultivar (Vitis labrusca). For the preparation of the extracts were used the dried leaves of gervão (Stachytarphetta cayenensis) and capim-limão (Cymbopogon citratus); fresh leaves of pau d'alho (Gallesia integrifolia) and wine residue (powder) from grape cultivar Isabel (Vitis labrusca). Each extract was applied at a concentration of $12 \%$ directly to the clusters 10 days before harvest and a portion of grapes received beyond the field application a new application in postharvest. The experimental design was randomized blocks in a factorial scheme with $5 \times 2$ (five treatments and pre-harvest and postharvest application) with four repetitions. Fruit quality parameters were assessed based on several criteria: color, $\mathrm{pH}$, titratable acidity, maturation index, level of phenolic compounds, anthocyanins and sensory analysis. It was found that the extracts were effective to maintain acidity, soluble solids, maturation index and sensory analysis. However, the application of lemongrass (Cymbopogon citratus) extract applied in pre- and postharvest treatments maintained $\mathrm{pH}$, chroma, anthocyanins levels and berries revealed a more intense color. The gervão (Stachytarphetta cayenensis) extract applied in pre- and postharvest kept a higher phenolic content when compared to the application only in pre-harvest. Pre- and postharvest application of lemongrass and gervão extracts showed to be a good alternative treatment for quality maintenance during storage of grapes.
\end{abstract}

Keywords: alternative treatment, storage, Vitis labrusca. 


\section{INTRODUÇÃO}

A uva cultivar Bordô (Vitis labrusca) destaca-se por sua elevada adaptação às condições climáticas do Brasil, apresenta boa fertilidade, tolerância a doenças de origem fúngica e é destinada, principalmente, à produção de sucos e vinhos. Sendo que, para a elaboração de subprodutos com qualidade adequada as práticas utilizadas na pré e pós-colheita são essenciais (Rizzon et al., 2010; Romanazzi et al., 2016).

Em uvas destinadas ao processamento, como a Bordô, valores adequados para $\mathrm{pH}$, acidez titulável, sólidos solúveis, compostos fenólicos e flavour no momento da colheita são fundamentais para a elaboração de sucos e vinhos com qualidade e boa aceitação entre os consumidores (Mota et al., 2009). Para isso, indica-se que a uva apresente $\mathrm{pH}$ entre 3,1 a 3,3; no mínimo 14ºrix e índice de maturação entre 15 a 45 (Brasil, 2004; Rizzon et al., 2004).

Para obtenção destas características físico-químicas necessita-se a adoção de medidas no controle de doenças e ao longo do armazenamento, as quais não deixem resíduos nos alimentos e sejam efetivos para manter a qualidade. Com isso, estudos apontam que derivados extraídos de plantas podem ser alternativas para minimizar as perdas quantitativas e qualitativas, devido às atividades antifúngicas e antioxidantes presentes (Di Scala et al., 2013; Souza, 2013).

É notável a grande diversidade de espécies de plantas no Brasil e a exploração da atividade biológica dos compostos secundários de extratos constituem potenciais métodos alternativos para controle de doenças em plantas devido às ações antibacteriana e antifúngica e ainda para manter a qualidade na pós-colheita (Rodrigues et al., 2005; Schwan-Estrada, 2009; Anaruma et al., 2010; Fonseca, 2012). Também, a vitivinicultura gera grande quantidade de resíduos, que em sua maioria são desperdiçados. Estima-se que, cerca de $13 \%$ do peso total da uva destinada ao processamento são descartados, esses resíduos são ricos em compostos biologicamente ativos, devido a presença de compostos fenólicos entre outros (Torres et al., 2002; Campos, 2005).

Abeywickrama et al. (2004) utilizaram extrato de capim-limão (Cymbopogon citratus) pulverizado em bananas para avaliar o controle de podridões (Colletotrichum musae, Lasiodiplodia theobromae e Fusarium proliferatum) e para a manutenção da qualidade pós-colheita. Observaram que a aplicação do extrato reduziu em $50 \%$ a severidade da doença. Para os frutos tratados foram encontradas parâmetros físicos e químicos, flavour e de aceitabilidade adequados.

Diante disso, destaca-se a necessidade de pesquisas com intuito de avaliar a utilização de diferentes extratos de plantas para qualidade pós-colheita de uvas. Portanto, o presente estudo buscou avaliar a eficácia da aplicação dos extratos de gervão (Stachytarphetta cayenensis), capim-limão (Cymbopogon citratus), pau d'alho (Gallesia integrifolia) e de resíduo de vinificação de uva cultivar Isabel (Vitis labrusca) na qualidade pós-colheita em uvas orgânicas de cultivar Bordô (Vitis labrusca).

\section{MATERIAIS E MÉTODOS}

\section{Localização}

O experimento foi realizado no mês de dezembro de 2014, em vinhedo comercial da cultivar Bordô, localizado no município de Marechal Cândido Rondon-PR, situado a $24^{\circ} 46^{\prime} \mathrm{S}$ e $54^{\circ} 22^{\prime} \mathrm{O}$. Solo tipo Latossolo roxo, topografia plana (Santos et al., 2006). Clima classificado em subtropical úmido mesotérmico, sendo que as temperaturas médias são de $20,6^{\circ} \mathrm{C}$ e $25^{\circ} \mathrm{C}$. As plantas foram cultivadas em sistema orgânico, enxertadas sobre o porta-enxerto 420 A (Vitis berlandieri x Vitis riparia), em sistema de espaldeiras, com espaçamento $1 \times 3 \mathrm{~m}$, parreiral com 13 anos de idade.

\section{Obtenção dos extratos}

Os extratos de gervão (Stachytarphetta cayenensis) e resíduo de uva foram obtidos por maceração da matéria seca, 24 g diluídas em $200 \mathrm{~mL}$ de água filtrada, aquecida a $60^{\circ} \mathrm{C}$. Os extratos capim-limão (Cymbopogon citratus) (matéria seca) e o pau d'alho (Gallesia integrifolia) (matéria fresca) foram obtidos por meio do extrato bruto aquoso. Todos os extratos foram utilizados na concentração de $12 \%$. As folhas de gervão (Stachytarphetta cayenensis) e capim-limão (Cymbopogon citratus) foram 
adquiridas em casas comerciais de especiarias. As folhas de pau d'alho (Gallesia integrifolia) foram coletadas no mês de dezembro de 2014 no Câmpus da Universidade Estadual de Maringá-PR e ficaram quatro dias em temperatura ambiente. Por sua vez, o resíduo de vinificação foi proveniente do resíduo de uva cultivar Isabel (Vitis labrusca) da vinícola localizada no município de Bituruna-PR.

\section{Delineamento experimental}

O delineamento experimental foi blocos ao acaso, com $5 \times 2$ (cinco tratamentos e dois aplicações, pré-colheita e pós-colheita) com quatro repetições. As uvas receberam uma pulverização dos extratos a $12 \%$ até o ponto de escorrimento (aproximadamente $10 \mathrm{~mL}$ por cacho) 10 dias antes da colheita no período da tarde. A colheita foi realizada quando os cachos apresentaram $19^{\circ}$ Brix.

As uvas foram transportadass para o laboratório de pós-colheita de frutas e hortaliças do Departamento de Agronomia da Universidade estadual do Centro-oeste-PR, onde as amostras foram divididas em dois grupos distintos: um mantido somente com a aplicação pré-colheita e a outro grupo de cachos recebeu uma nova aplicação dos tratamentos (aplicação pós-colheita) com o uso de embalagem de algodão recoberta com papel manteiga (formato de um envelope), tamanho de $10 \mathrm{~cm}$ cada e foram autoclavados antes de receberem os tratamentos. As embalagens foram umedecidas com os extratos aquosos, $10 \mathrm{~mL}$ de cada extrato e o tratamento testemunha recebeu apenas água destilada, colocados sob a bandeja e fechados com embalagens plásticas.

Cada bandeja, identificada, contendo três cachos foi mantida em câmara de crescimento, tipo BOD, no escuro e com temperatura $4 \pm 2{ }^{\circ} \mathrm{C}$ até a data de avaliação, na qual ainda as uvas apresentavam características adequadas para a comercialização.

Foram analisadas as características físico-químicas dos cachos, para isso foram utilizadas as seguintes variáveis: $\mathrm{pH}$ (determinado por meio da medição direta por potenciometria); acidez titulável expressa em $\mathrm{mg}$ de ácido tartárico (Instituto Adolfo Lutz, 1985); sólidos solúveis (avaliados por refractometria); índice de maturação (relação de sólidos solúveis com a acidez titulável, SST/ AT). A cor foi avaliada por colorimetria e foram obtidos os seguintes parâmetros: luminosidade, ângulo Hue e croma, as leituras realizadas no sistema $L^{*} a^{*} b^{*}$, na qual $L^{*}$ indicou a luminosidade, $a^{*}$ e $b^{*}$ cromaticidade (Glossary, 2007). O teor em compostos fenólicos foi avaliado por espectrofotometria (Rossi e Sinleton, 1965); e o teor em antocianinas foi avaliado por diferencial de $\mathrm{pH}$ (Guiusti e Ronald, 2001). A análise sensorial foi realizada utilizando a escala hedônica composta por notas de 1 a 9 , o procedimento foi previamente avaliado e aprovado sob o parecer 896.236 do comitê de ética em pesquisa com seres humanos.

Os dados obtidos foram submetidos à análise de variância e quando significativo aplicou-se o teste de comparação de médias Tukey $(\mathrm{p}<0.05)$, utilizando o programa estatístico SISVAR versão 5.1 (Ferreira, 2007).

\section{RESULTADOS E DISCUSSÃO}

$\mathrm{Na}$ avaliação 13 dias após tratamento no campo observou-se uma influência significativa de todos os fatores analisados para a variável $\mathrm{pH}$, observando-se que todos os extratos aplicados diferiram do tratamento testemunha. A aplicação do extrato de capim limão (Cymbopogon citratus) na pré-colheita e a aplicação do extrato de pau d'alho (Gallesiaintegrifolia)napréenapós-colheitaocasionou os maiores valores para esta variável (Figura 1).

A aplicação pré-colheita do extrato pau d'alho (Gallesia integrifolia) foi eficiente na manutenção do $\mathrm{pH}$ dentro da faixa adequada para a cultivar, sendo que os demais tratamentos aumentaram, consideravelmente, o valor para esta variável. Daudt e Fogaça (2008), ressaltam que um valor alto de $\mathrm{pH}$ na uva pode comprometer a conservação, reduzir a cor e pode comprometer a qualidade microbiológica. No entanto, observou-se que o extrato de capim-limão (Cymbopogon citratus) aplicado na pré e pós-colheita também foi eficaz para manter o potencial hidrogeniônico $(\mathrm{pH})$ da uva cv. Bordô. Resultados semelhantes a este foram obtidos por Abeywickrama et al. (2004), que verificaram que o extrato de capim-limão (Cymbopogon citratus) pulverizado no momento da colheita não influenciou o $\mathrm{pH}$ em frutos de bananas (Musa acuminata $\mathrm{AAB}$ ). 


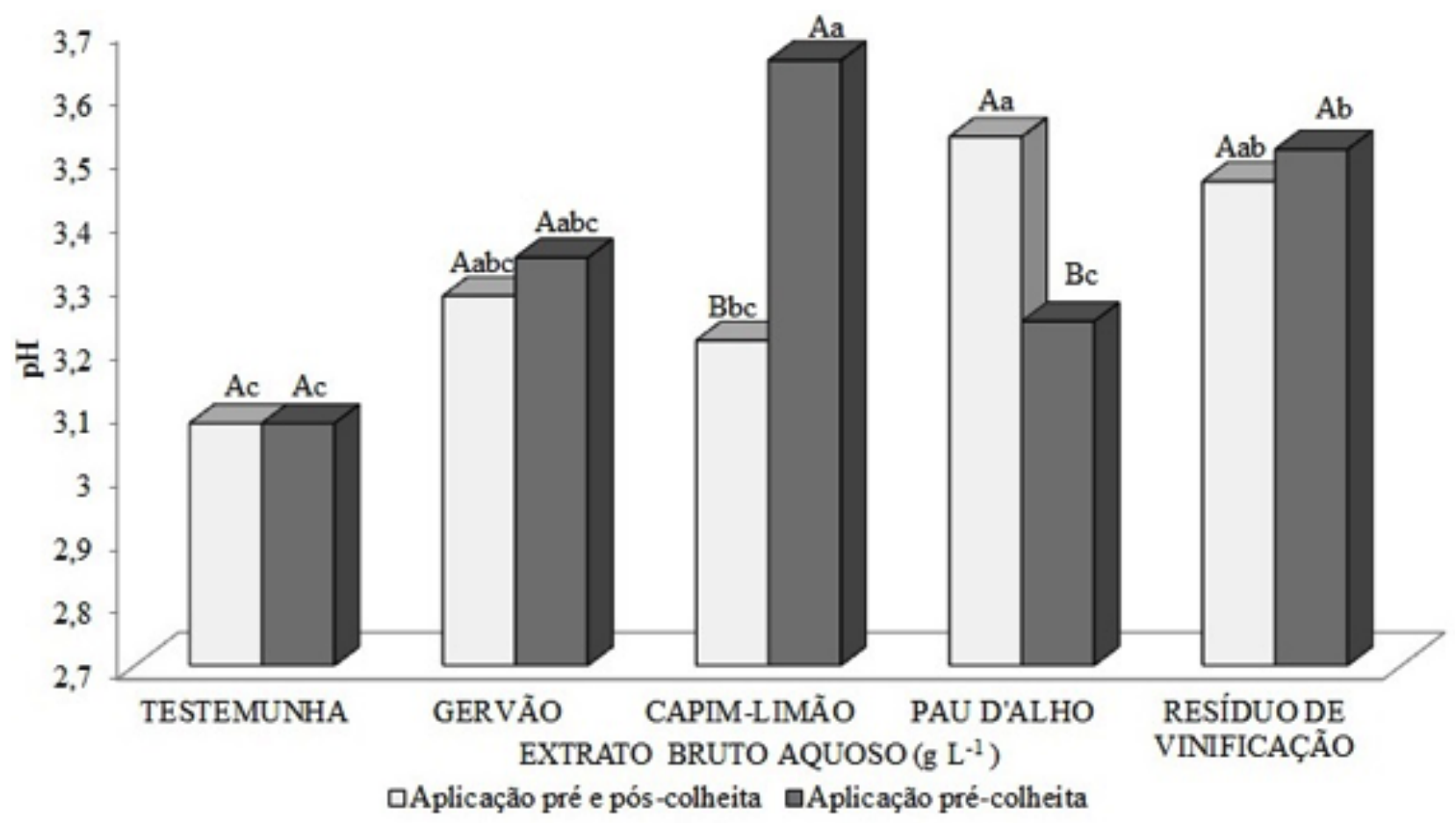

Figura 1 - pH em uva cultivar Bordô tratada com diferentes extratos vegetais aplicados na pré-colheita. Coeficiente de variação dos dados (CV): 12,39\% (A); CV: 4,47\% (B). Letras iguais não diferem entre si pelo teste de Tukey $(p<0.05)$.

Para a variável luminosidade os diferentes extratos aplicados influenciaram significativamente este parâmetro. Os cachos com aplicação do extrato de gervão (Stachytarphetta cayenensis) apresentaram menor valor para esta variável, diferindo do tratamento testemunha (Figura 2).
Observou-se o efeito dos diferentes extratos vegetais aplicados para a variável ângulo Hue, o extrato de capim-limão (Cymbopogon citratus) proporcionou maior valor para esta variável e diferiu do tratamento testemunha (Figura 3A). A aplicação pré-colheita também proporcionou maior valor

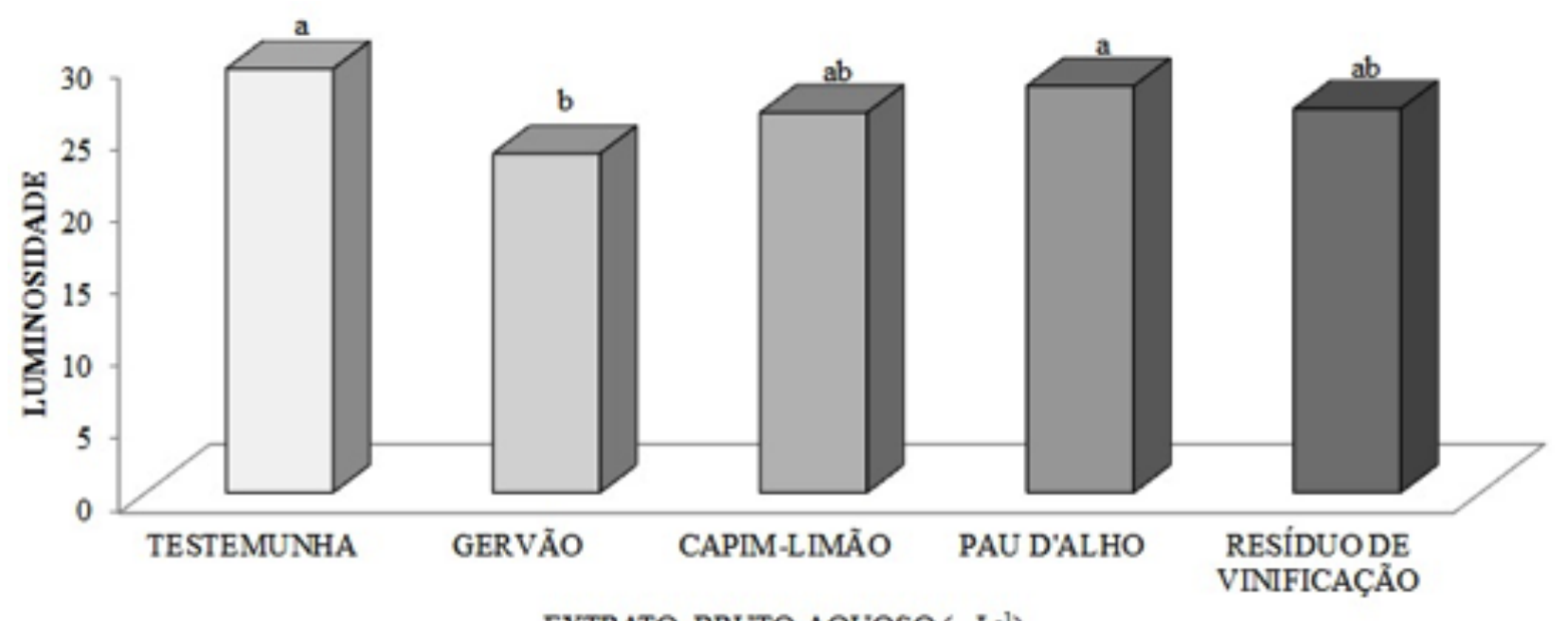

EXTRATO BRUTO AQUOSO $(\mathrm{g} \mathrm{L}-1)$

Figura 2 - Luminosidade em uva cultivar Bordô tratadas com diferentes extratos vegetais. CV: 9,62\% (A e B). Letras iguais não diferem entre si pelo teste de Tukey $(p<0.05)$. 
para esta variável, quando comparadas à aplicação pré e pós-colheita (Figura 3B).

Para o parâmetro croma, não houve interação entre os fatores, contudo, observou-se diferença significativa entre os diferentes extratos aplicados, nos cachos os quais receberam a aplicação do extrato de capim-limão (Cymbopogon citratus) o valor foi superior para esta variável quando comparada aos demais tratamentos e testemunha (Figura 4).
Em relação à coloração os valores observados para as variáveis ângulo Hue e croma em uvas tratadas com diferentes extratos vegetais indicam um efeito na coloração o qual acarretou bagas com cor roxa em tonalidade mais acentuada e com maior saturação (Glossary, 2007), desta forma, infere-se que a aplicação dos extratos na pré-colheita e o extrato de capim-limão (Cymbopogon citratus) proporcionaram aos cachos uma coloração acentuada roxa.

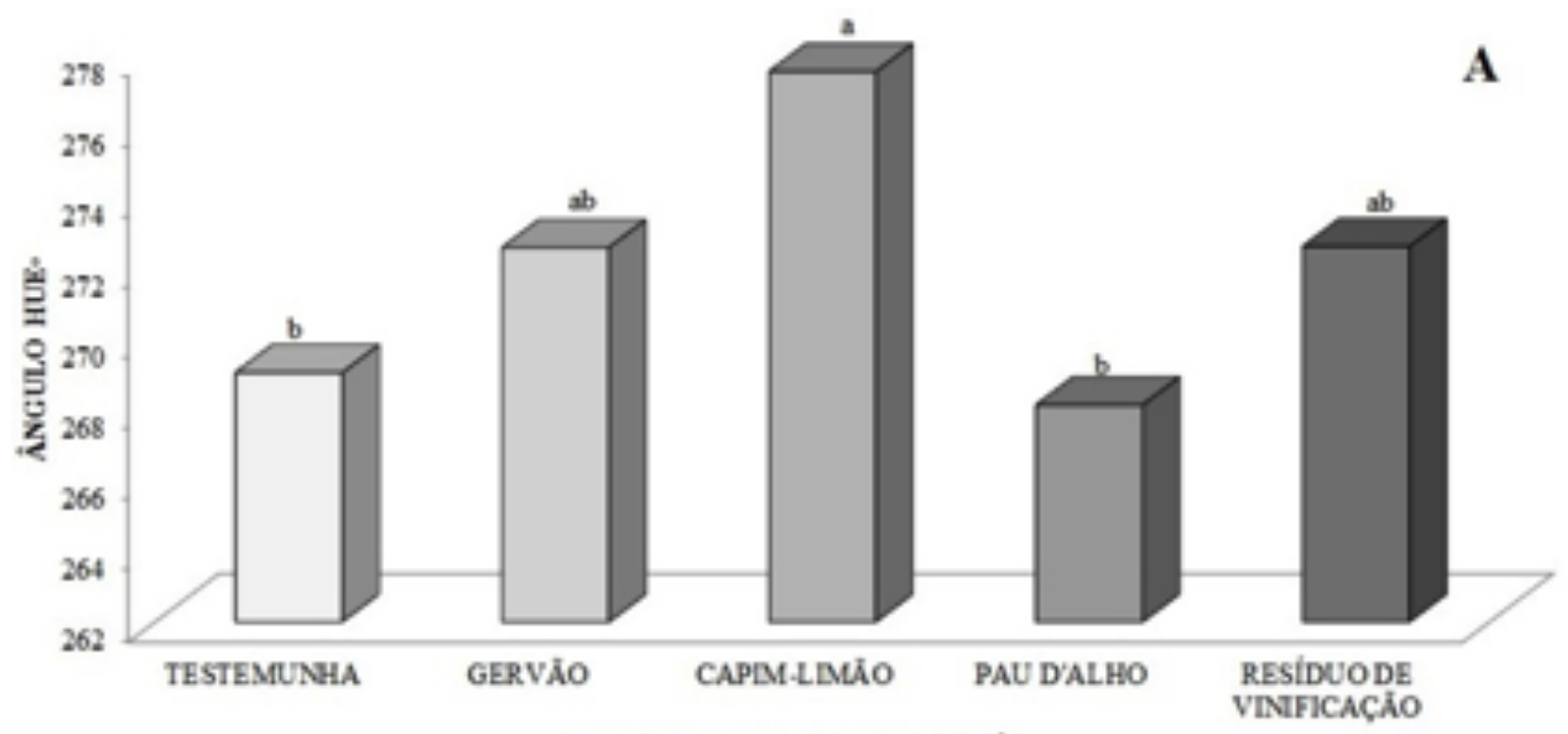

EXTRATO BRUTO AQUOSO (g L $\left.\mathrm{L}^{-4}\right)$

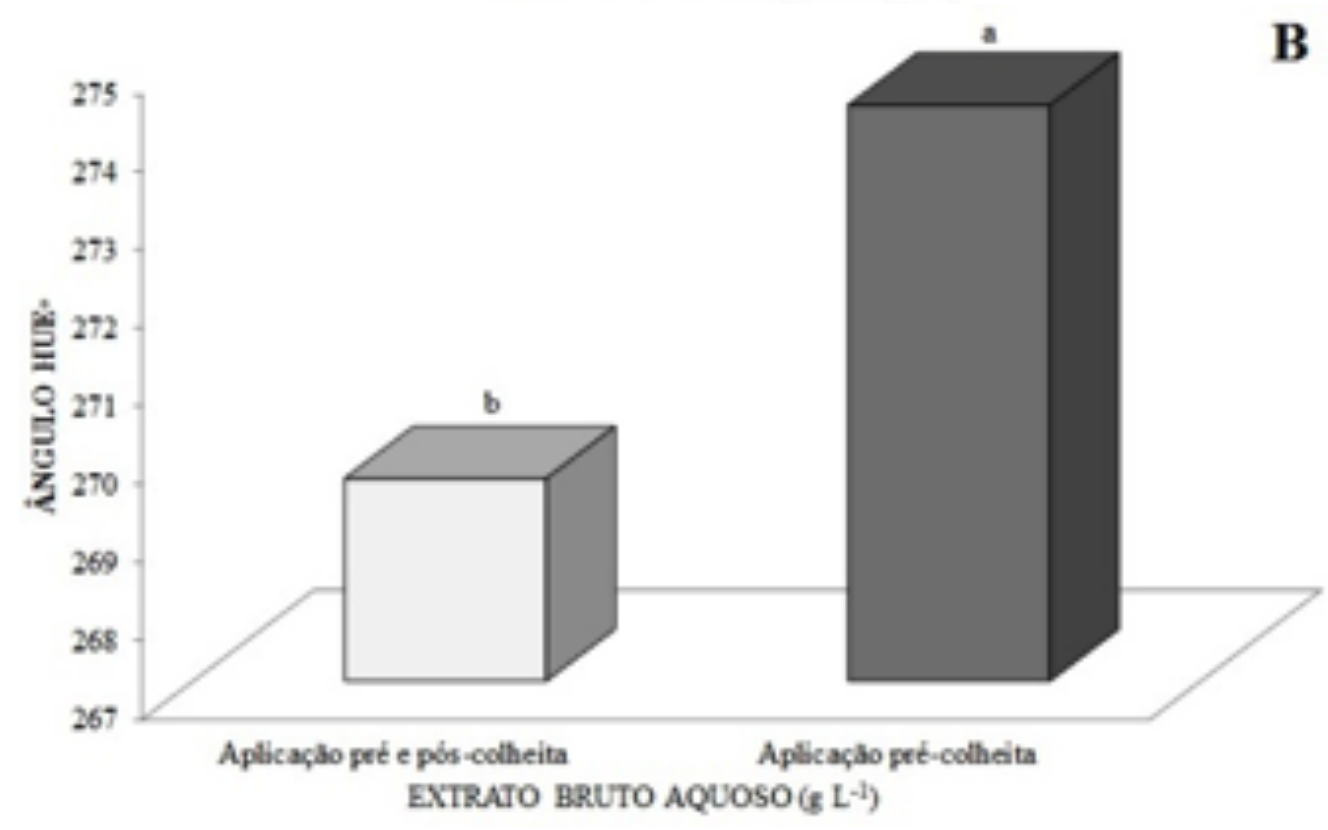

Figura 3 - Ângulo Hue em uva cultivar Bordô tratadas com diferentes extratos na pré e pós-colheita (A e B). CV: 2,25\% (A e B). Letras iguais não diferem entre si pelo teste de Tukey $(p<0.05)$. 


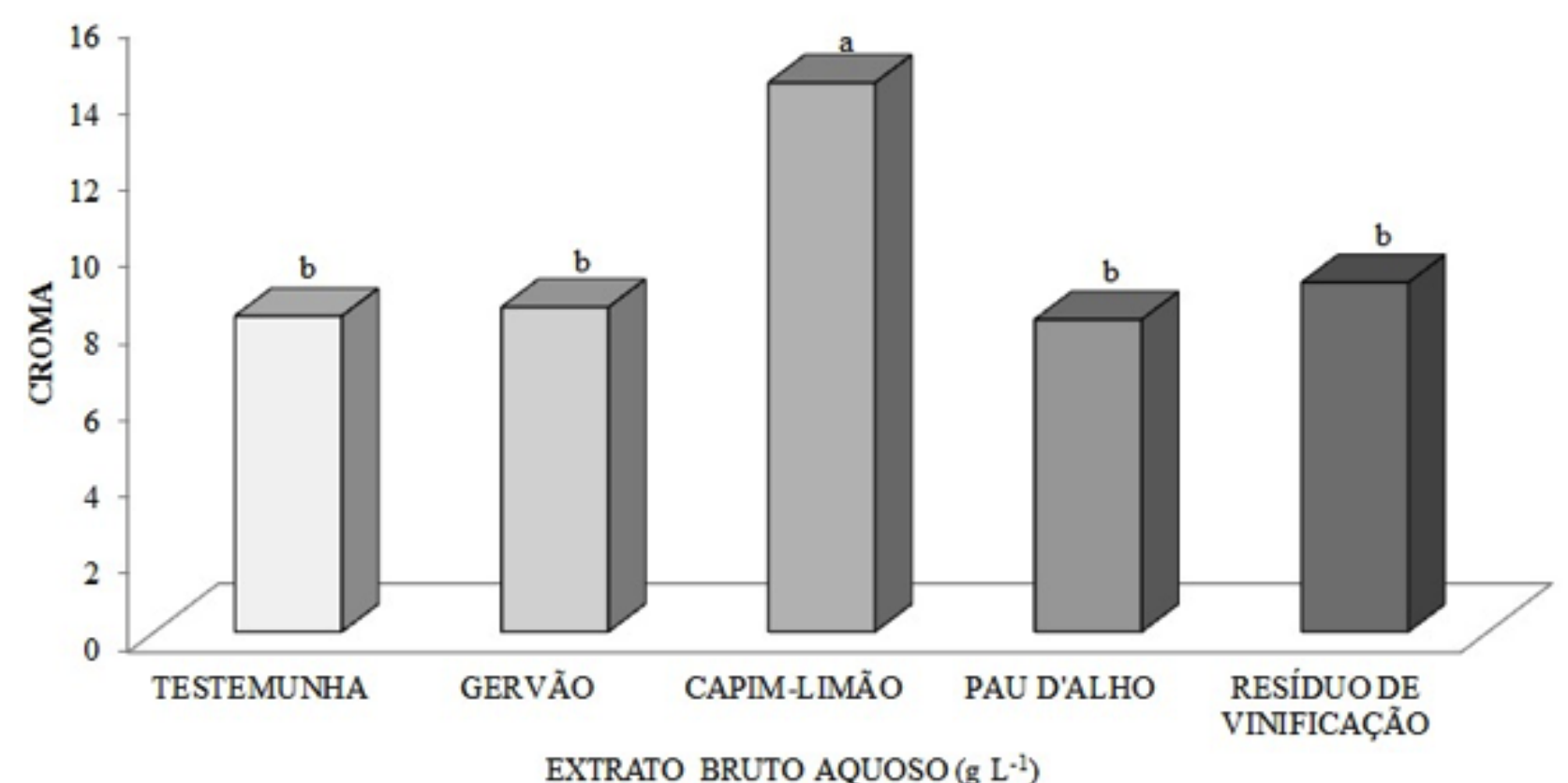

Figura 4 - Croma em uva cultivar Bordô tratadas com diferentes extratos vegetais. CV: 36,59\% (A); CV: 27,00\% (B). Letras iguais não diferem entre si pelo teste de Tukey $(p<0.05)$.

Como já citado, observou-se que a aplicação do extrato de gervão (Stachytarphetta cayenensis) acarretou menor valor para a variável luminosidade, o extrato de capim-limão (Cymbopogon citratus) maior valor para as variáveis ângulo Hue e croma em cachos de uva cultivar Bordô tratadas com diferentes extratos. Com isso, indica-se que os extratos foram eficazes para intensificar a coloração na baga. A coloração intensa, a qual é característica da cultivar permite que ela seja adicionada na indústria a sucos de outras cultivares, as quais apresentam coloração menos intensa, a fim de ressaltar a cor e melhorar os atributos sensoriais (Embrapa, 2010).

$\mathrm{Na}$ avaliação do teor em compostos fenólicos, a aplicação pré e pós-colheita do extrato de gervão (Stachytarphetta cayenensis) conferiu aos cachos valor superior quando comparado à aplicação do mesmo extrato somente na pré-colheita. A aplicação dos demais extratos não ocasionou diferenças significativas (Figura 5).

Os extratos vegetais utilizados no presente estudo proporcionaram aos cachos maior concentração de $\mathrm{mg}$ de ácido gálico $\mathrm{g}^{1} \mathrm{e} \mathrm{mg}$ de antocianina $\mathrm{g}^{1}$. Estes atributos são fundamentais para a coloração, capacidade antioxidante e proteção em uvas (Muñoz-Espada et al., 2004; Naczk e Shahidi, 2004). Há poucos relatos na literatura na utilização de extratos vegetais em pós-colheitas de frutas, entretanto, Xu et al. (2007) estudando o efeito do extrato da semente de uva na qualidade de uva cv. Redglobe e Feliziani et al. (2015) trabalhando com extratos vegetais Abies spp. e Polygonum spp em morangos, ambos os autores verificaram a manutenção da qualidade dos frutos resultante da aplicação dos extratos vegetais, sugerindo uma ação antifúngica e antioxidante.

$\mathrm{Na}$ avaliação das antocianinas foi observado um efeito significativo da aplicação dos extratos vegetais. Assim a aplicação pré-colheita e pós-colheita dos extratos de gervão (Stachytarphetta cayenensis) e de capim-limão (Cymbopogon citratus) em cachos proporcionou maiorvalor paraesta variável, quando comparado a aplicação destes extratos somente na pré-colheita, sendo que um valor maior atribuído para as antocianinas corresponde a coloração em intensidade mais próxima ao roxo (Figura 6).

Com bases nos resultados obtidos, verificou-se que os extratos aquosos capim-limão (Cymbopogon citratus) e gervão (Stachytarphetta cayenensis) foram eficazes com aplicação pré e pós-colheita, 


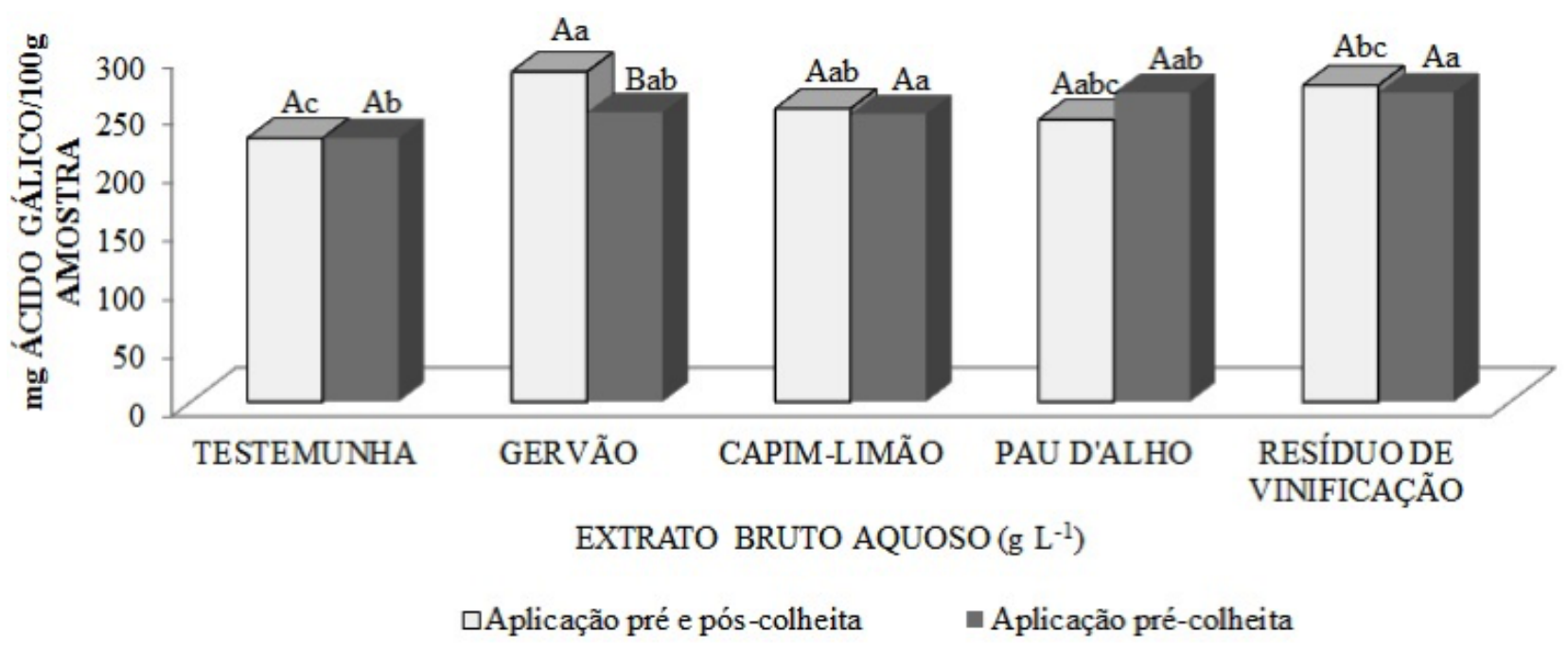

Figura 5 - Compostos fenólicos em uva cultivar Bordô tratadas com diferentes extratos vegetais. Letras maiúsculas comparam a aplicação, letras minúsculas comparam os tratamentos. CV: 4,92\% (A); CV: 6,93\% (B). Letras iguais não diferem entre si pelo teste de Tukey $(p<0.05)$.

aumentando o conteúdo de compostos fenólicos e antocianinas em uvas cv. Bordô, o que influencia a qualidade dos produtos finais provenientes do processamento, assim, tornam-se alternativas viáveis para a agricultura orgânica.
Para a acidez titulável, os sólidos solúveis e o índice de maturação e análise sensorial, a aplicação de extratos vegetais não mostraram efeitos significativos, as médias encontradas foram: $0,74 \mathrm{mg}$ de ácido tartárico g1 , 20Brix, 40 e 7 ("gostei moderadamente").

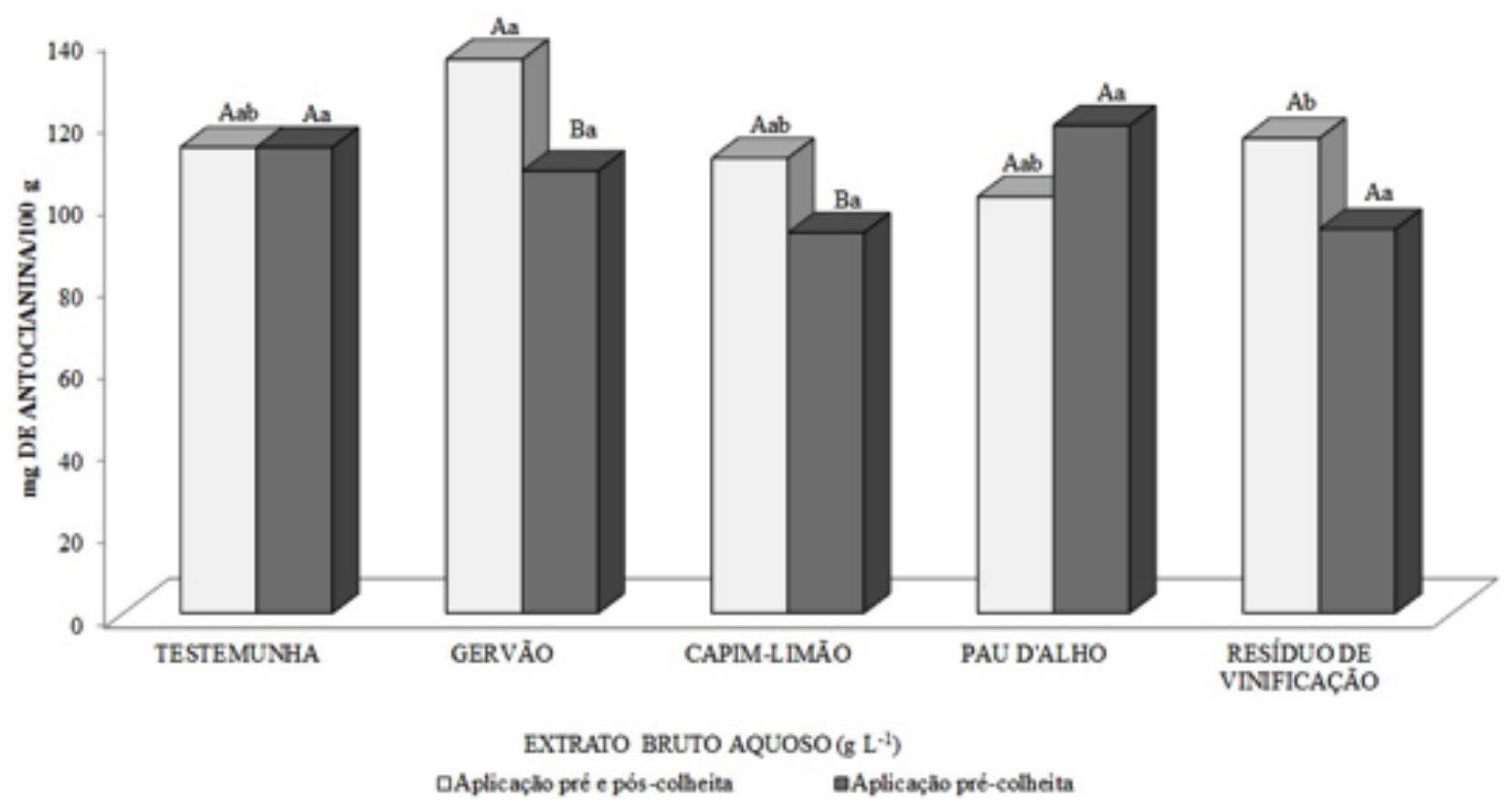

Figura 6 - Antocianina em uva cultivar Bordô com aplicação pré-colheita (A) e pré e pós-colheita de extratos vegetais, 60 horas (B) após a aplicação na pós-colheita. Letras maiúsculas comparam a aplicação, letras minúsculas comparam os tratamentos. CV: 9,20\% (A); CV: 17,61\% (B); CV\%: 11,92 (C). Letras iguais não diferem entre si pelo teste de Tukey $(p<0.05)$. 
No presente estudo, observou-se que todos os parâmetros de qualidade avaliados estavam dentro do padrão estabelecidos como ideais para a cultivar Bordô. De acordo com Rizzon et al. (2000), o mínimo de sólidos solúveis deve ser de $14 \%$, sendo que se considera uma variação até $17 \%$ no momento da colheita para esta cultivar. Para o índice de maturação indica-se o valor de 40 a 45 (Rizzon e Menuguzzo, 2007), sendo que o índice de maturação é um importante critério para avaliar a qualidade em uvas, e reflete o potencial para produção de sucos e uvas de qualidade, referindo-se ao balanço entre o açúcar e a acidez presentes. Este atributo confere o equilíbrio gustativo e determina a comercialização (Daudt e Fogaça, 2008).

\section{CONCLUSÃO}

A aplicação pré-colheita do extrato de pau d'alho (Gallesia integrifolia) foi eficiente para manter o $\mathrm{pH}$ dentro da faixa estabelecida como ideal para a cultura.

O extrato de gervão (Stachytarphetta cayenensis) influenciou a coloração, conteúdo de compostos fenólicos e de antocianinas.

Os cachos tratados em pré e pós-colheita com extrato de capim-limão (Cymbopogon citratus), apresentaram uma coloração mais intensa, mantendo-se o potencial hidrogeniônico.

\section{REFERÊNCIAS BIBLIOGRÁFICAS}

Abeywickrama, K.; Kularathna, L.; Sarananda, K. \& Abeygunawardena, D. (2004) - Cymbopogon citratus (lemongrass) and citral $\mathrm{a}+\mathrm{b}$ spray treatments alone or in combination with sodium bicarbonate in controlling crown rot in embul banana (Musa acuminata AAB). Tropical Agricultural Research and Extension, vol. 7.

Anaruma, N.D.; Schmidt, F.L.; Duarte, M.C.T.; Figueira, G.M.; Delarmelina, C.; Benato, L.A. \& Sartoratto, A. (2010) - Control of Colletotrichum gloeosporioides (Penz) Sacc. Brazilian Journal of Microbiology, vol. 41, n. 1, p. 66-73. http://dx.doi.org/10.1590/S1517-83822010000100012

BRASIL. (2004) - Secretaria de Políticas Agrícolas, Ministério da Agricultura. [cit. 2015.09.05]. http://www.agricultura.gov.br.

Campos, L.M.A.S. (2005) - Obtenção de extratos de bagaço de uva Cabernet Sauvignon (Vitis vinífera): Parâmetros de processos e modelagem matemática. Dissertação (Mestrado em Engenharia de alimentos). Universidade Federal de Santa Catarina, Florianópolis, 123 p.

Daudt, C.E. \& Fogaça, A.O. (2008) - Efeito do ácido tartárico nos valores de potássio, acidez titulável e pH durante a vinificação de uvas Cabernet Sauvignon. Ciência Rural, vol. 38, n. 8, p. 2345-2350. http://dx.doi. org/10.1590/S0103-84782008000800039

Di Scala, K.; Vega-Gálvez, A.; Ah-Hen, K.; Nuñez-Mancilla, Y.; Tabilo-Munizaga, G.; Pérez-Won, M.E. \& Giovagnoli, C. (2013) - Chemical and physical properties of aloe vera (Aloe barbadensis Miller) gel stored after high hydrostatic pressure processing. Food Science and Technology, vol. 33, n. 1, p. 52-59.

EMBRAPA. (2010) - Centro nacional de pesquisa em uva e vinho. Dados da viticultura: banco de dados de uva, vinho e derivados. EMPRESA BRASILEIRA DE PESQUISA AGROPECUÁRIA, Brasília. [cit. 2015.08.05]. http://www.enpuv.embrapa.br

Feliziani, E.; Landi, L. \& Romanazzi, G. (2015) - Preharvest treatments with chitosan and other alternatives to conventional fungicides to control postharvest decay of strawberry. Carbohydrate Polymers, vol. 132, p. 111-117. http://dx.doi.org/10.1016/j.carbpol.2015.05.078

Ferreira, D.F. (2007) - Sisvar: sistema de análise de variância para dados balanceados, versão 5.1. DEX/ UFLA, Lavras. Software estatístico.

Fonseca, M.C.M. (2012) - Pesquisa, produção de plantas medicinais para aplicação no Sistema Único de Saúde. Espaço para o produtor, EPAMIG, Viçosa.

Giusti, M. \& Ronald, E. (2001) - Current protocols (Characterization and Measurement of Anthocyanins by UV-Visible Spectroscopy). Wrolstad.

Glossary, C. (2007) - Precise color communication: Color control form perception to instrumentation. [cit. 2015.05.23]. http://www.konicaminolta.com 
Instituto Adolfo Lutz (1985) - Normas Analíticas do Instituto Adolfo Lutz. Métodos químicos e físico para análise de alimentos. vol. 1, 3. ․ Ed., p.533, São Paulo.

Mota, R.V. da; Souza, C.R. de; Favero, A.C.; Silva, C.P.C.; Carmo, E.L. do; Fonseca, A.R. \& Regina, M. de A. (2009) - Produtividade e composição físicoquímica de bagas de cultivares de uva em distintos portaenxertos. Pesquisa Agropecuária Brasileira, vol. 44, n. 6, p.576582. http://dx.doi.org/10.1590/S0100-204X2009000600005

Muñoz-Espada, A.C.; Wood, K.V.; Bordelon, B. \& Watkins, B.A. (2005) - Anthocyanin quantification and radical scavenging capcity of Concord, Norton, and Marechal Foch grapes and wines. Journal of Agriculture and Food Chemistry, vol. 52, n. 22, p. 6779-6786. http://dx.doi.org/10.1021/ffo40087y

Naczk, M. \& Shaihidi, F. (2004) - Extraction and analysis of phenolics in food. Journal of Chromatography A, vol. 1054, n. 1-2, p. 95-111. http://dx.doi.org/10.1016/j.chroma.2004.08.059

Rizzon, L.A. \& Meneguzzo, J. (2007) - Suco de uva. Embrapa Informação Tecnológica, Brasília.

Rizzon, L.A.; Meneguzzo, J. \& Manfroi, L. (2004) - Processamento de uva, vinho tinto, graspa e vinagre. Embrapa Informação Tecnológica, Brasília.

Rizzon, L.A.; Miele, A. \& Meneguzzo, J. (2000) - Avaliação da uva cv. Bordô para a elaboração de vinho tinto. Ciência e Tecnologia de Alimentos, vol. 20, n. 1, p. 115-121. http://dx.doi.org/10.1590/S0101-20612000000100022

Rodrigues, F.A.; Jurick, W.M.; Datnoff, L.E.; Jones, J.B. \& Rollins, J.A. (2005) - Silicon influences cytological and molecular events in compatible and incompatible rice-Magnaporthe grisea interactions. Physiological and Molecular Plant Pathology, vol. 66, n. 4, p. 144-159. http://dx.doi.org/10.1016/i.pmpp.2005.06.002

Romanazzi, G.; Smilanick, J.L.; Feliziani, E. \& Droby, S. (2016) - Integrated management of postharvest gray mold on fruit crops. Postharvest Biology and Technology, vol. 113, p. 69-76. http://dx.doi.org/10.1016/j. postharvbio.2015.11.003

Rossi, J.A. \& Sinleton, V.L. (1965) - Colorimetry of total phenolics with phosphomolybdic-phosphotungstic acid reagents. American Journal of Enology and Viticulture, vol. 16, p. 144-158.

Santos, H.G.; Jacomine, P.K.T.; Anjos, L D.; Oliveira, V.D.; Oliveira, J.D.; Coelho, M.R. \& Cunha, T.D. (2006) - Sistema brasileiro de classificação de solos. [cit. 2015.09.25]. http://www.agrolink.com.br

Souza, J.L. (2013) - Pré-colheita e colheita de uvas na Serra gaúcha (Cooperativa Vinícola Aurora de Bento Gonçalves/ RS) e Legislação de bebidas derivadas da uva e do 83 vinho no MAPA-SC. Trabalho de conclusão, Universidade Federal de Santa Catarina, Florianópolis, SC. 52 p.

Schwan-Estrada, K.R.F. (2009) - Extratos vegetais e de cogumelos no controle de doenças de plantas. Horticultura Brasileira, vol. 27, n. 2, S4038-S4045.

Torres, J.L.; Varela, B.; García, M.T.; Carilla, J.; Matito, C.; Centelles, J.J.; Cascante, C.; Sort, X. \& Bobet, R. (2002) - Valorization of grape (Vitis vinifera) by products. Antioxidant and biological properties of polyphenolic fractions differing in procyanidin composition and flavonol content. Journal of Agricultural and Food Chemistry, vol. 50, n. 26, p. 7548-7555. http://dx.doi.org/10.1021/ff025868i

Xu, W.T.; Huang, K.L; Guo, F.; Qu, W.; Yang, J.J.; Liang, Z.H. \& Luo, Y.B. (2007) - Postharvest grapefruit seed extract and chitosan treatments of table grapes to control Botrytis cinerea. Postharvest Biology and Technology, vol. 46, n. 1, p. 86-94. http://dx.doi.org/10.1016/j.postharvbio.2007.03.019 